\title{
NITESat: A High Resolution, Full-Color, Light Pollution Imaging Satellite Mission
}

\author{
Ken Walczak ${ }^{1 *}$, Geza Gyuk ${ }^{1}$, Andrew Kruger ${ }^{2}$, Enoch Byers², Sigi Huerta ${ }^{2}$ \\ ${ }^{1}$ Adler Planetarium, Chicago, Illinois 60605, USA \\ ${ }^{2}$ City Colleges of Chicago, Chicago, IL USA
}

Received 16 December 2016, Accepted 08 May 2017

\begin{abstract}
The NITESat (Night Imaging and Tracking Experiment Satellite) mission is a 2U CubeSat satellite designed for nighttime Earth imaging to quantify and characterize light pollution across the Midwestern United States. It is accompanied and supported by an array of ground-based light pollution observing stations called GONet (Ground Observing Network). NITESat is a pilot mission testing the potential for a simple and inexpensive $(<\$ 500,000)$ satellite to deliver high-resolution, three-color regional data of artificial light at night. In addition, GONet will form the core of an educational outreach program by establishing an array of all-sky monitors covering the imaging region of the satellite with $20+$ full sky light pollution citizen-operated stations. This will provide synchronized data coinciding with the NITESat overpasses as well as providing near continuous night sky quality monitoring. If the initial mission is a success, the potential exists to expand the program into a low cost constellation of satellites capable of delivering global coverage. NITESat is being designed, built and will be operated by the Far Horizons program at the Adler Planetarium in Chicago, Illinois. Far Horizons is a student and volunteer centered program offering hands-on engineering and scientific research opportunities for education.
\end{abstract}

Keywords: NITESat, GONet, CubeSat, Remote sensing, Satellite, Light pollution

\section{Background}

Light Pollution (LP) by its simplest definition is the contamination of the natural nighttime environment by light of anthropomorphic origin. Many studies over the past decades have shined a light on the impacts and costs of light pollution. Much of the valuable data for light pollution research beyond local measurements has come from orbital satellite missions.

Studies have shown that excessive LP can adversely impact human health [1], animal behavior and even entire ecological systems [2] among other negative impacts. It is estimated $60 \%$ of the residents of Europe and $80 \%$ of North American cannot see the Milky Way from their homes due to light pollution [3]. This not only reduces the innate understanding and connection we have to the Universe but it robs us of context of our place within it.

Light pollution can also have economic implications: artificial light reaching space is wasted energy. The financial cost of this waste can be quantified by careful observations from space. As an example, an analysis of 2008 data shows that due to poor lighting practices and antiquated fixtures Chicago, a city of 2.7 million, spends an estimated 30\% (\$5,400,000 USD) of its annual \$18M USD public lighting budget solely in light ineffectively sent up into the atmosphere [4]. This estimate is based on an inventory of Chicago lighting fixtures and assumed efficiencies of those fixtures. Space-based observations could help give a more precise figure of those assumptions

*Walczak K, E-mail address: kwalczak@adlerplanetarium.org 
by measuring actual flux. Beyond the obvious financial burden created by this inefficiency, the energy required to generate this light - in most cases by the burning of fossil fuels - has direct and avoidable environmental repercussions.

As awareness grows, preconceptions can begin to fade. For example, much of the public believes the quantity of light illuminating their nighttime environment correlates directly with safety. At least one study has cast doubt on this assumption [5]. As researchers uncover relationships between LP and our environment the public is, in turn, gaining an awareness of its impact on their lives. Much of the growth in knowledge of the effects of light pollution on biology, health, economy and ecology can be attributed to the availability of large data sets quantifying LP from orbital satellite missions. The information provided by these large government-funded missions has given researchers an opportunity to understand LP on a global and regional level by quantifying LP emission.

Since 1973 the Optical Linescan System (OLS) instrument has been flown on board a continuous series of U.S. Air Force Defense Meteorological Satellite Program (DMSP) missions. Once the data products from the OLS were made publicly available in 1992, those interested in LP research for the first time benefited from full global maps of LP. The OLS data helped expand the field of LP research [6]. Although the OLS data provided much valuable information, there are significant limitations for researchers. Among the limitations from the OLS instrument are; a limited 5km/px resolution, a less than favorable 19:30 local time overpass time, oversaturation in urban areas, single panchromatic bandpass and no calibration [7].

Beginning at the end of 2011, the Suomi National Polar-orbiting Partnership (Suomi-NPP) satellite was launched. It carries the Visible Infrared Imaging Radiometer Suite (VIIRS) instrument. The VIIRS Day Night Band (DNB) data has addressed some of the shortcomings of the OLS data. Although the data provided by the Suomi-NPP satellite has increased researchers' ability to quantify LP, limitations still remain. Some of these limitations include: $742 \mathrm{~m} / \mathrm{px}$ resolution, a late 1:30AM local time overpass time, a single panchromatic bandpass imagery [8] and a "blue blindness" above 500nm that fails to detect significant emission from many styles of outdoor LED lighting [3].

\section{Mission Overview and Goals}

The Night Imaging and Tracking Experiment Satellite (NITESat) mission aims to improve the quality of LP data available from orbit. The mission consists of two primary components: NITESat and GONet. NITEsat is an orbital nanosatellite with a sensitive, high resolution, RGB imaging system as a primary payload. The Ground Observing Network (GONet) is an array of ground-based, all-sky imaging stations. Both systems work in tandem to validate and support the data products produced by the mission. NITESat and GONet have been specifically designed to address many of the shortcoming of presently available on-orbit data as well as exploring the future of LP observations with inexpensive nanosatellites.

To achieve this objective, the design of the NITESat mission is driven by the following goals:

\subsection{Produce a complete regional map of LP}

The NITESat mission will produce a complete map of LP across a significantly large region centered on the city of Chicago. The initial mission goal is to image a 1000x1000km area centered on Chicago (see Figure 1) multiple times throughout the mission lifetime of one to two years. Centering on Chicago (where the mission is being designed and operated) will allow regional coverage of a diverse range of LP affected environments from dense urban centers through to relatively unaffected unpopulated areas. An additional advantage of imaging this area is that it contains a large expanse of relatively pristine dark locations over the two Great Lakes (Michigan and Huron). This allows the opportunity to use it as a low-level calibration source. The size and location of this area also allows realistic and effective distribution and maintenance of the GONet system for on-orbit to ground correlation.

The primary need to limit the imaging footprint is due to the low downlink capacity of the single Ground Station based at the Adler Planetarium. Mission performance studies suggest that the addition of other Ground Stations run by partnering institutions across the world, will allow expansion of the imaging opportunities in nearly direct proportion to the available downlink capacity (up to a maximum of 10-20 fold increase). 


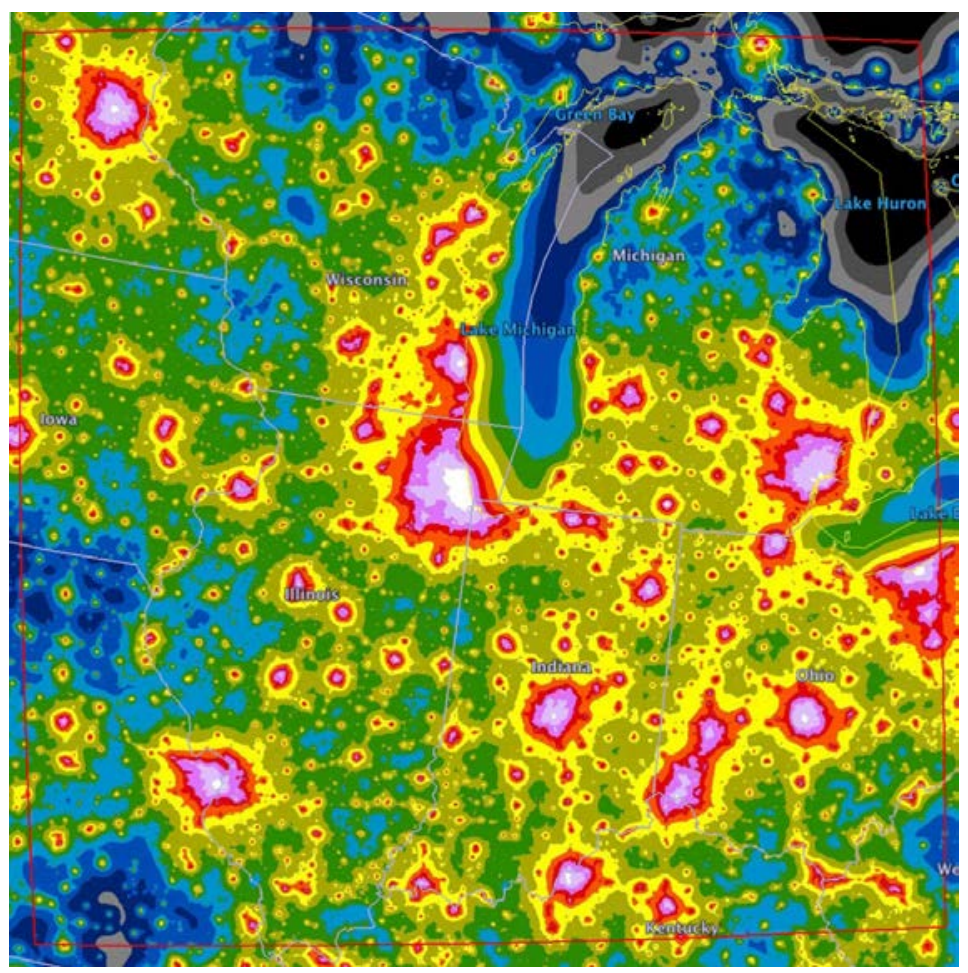

Fig. 1. Light pollution intensity map for the 1000x1000km NITESat imaging area centered on Chicago. This region covers 4 orders of magnitude in population density from rural to one of the world's most light-polluted cities. The Great Lakes provide areas of contrasting zero population. Image credit: F. Falchi, et al 2016 [3]

\subsection{Collect color information to distinguish LP sources}

NITESat imagery will provide color information sufficient enough to distinguish between major types of lighting used in municipal and commercial nighttime settings. NITESat will be equipped with an RGB imaging system. With this data, major types of light sources (ex., HPS, MV and most LED) will be distinguishable from each other [9]. Surveys of specific artificial lighting environments across the imaging footprint will be achievable. Direct analysis of comparable communities utilizing different types of artificial light can also be made.

\subsection{Improve on the resolution from VIIRS data}

Improved resolution compared to VIIRS data will add to our understanding of LP sources and effects on a smaller, more defined scale. The present design of the mission will achieve a GSD resolution of 100m/px, a more than 7-fold increase over the VIIRS data. At this resolution, small-scale variations of artificial lighting can be distinguished. A simulation of the expected results combining resolution and RGB information of NITESat versus VIIRS data can be seen in Fig. 2

\subsection{Provide synchronized ground-to-orbit data}

The NITESat mission will be flown in conjunction with an array of ground-based observing stations. These GONet stations will provide ground-truthing for the on-orbit NITESat data. By synchronizing the GONet images precisely with the timing of NITESat overpasses, ground-to-orbit correlations can be created. This data can be valuable in modeling atmospheric scatter, aerosol distribution and other parameters important for an understanding of LP.. Also, select GONet nodes will be equipped with a high-intensity, calibrated light source 
to be triggered on select NITESat overpasses. These sources will be designed to perform absolute calibration for the NITESat imaging system.
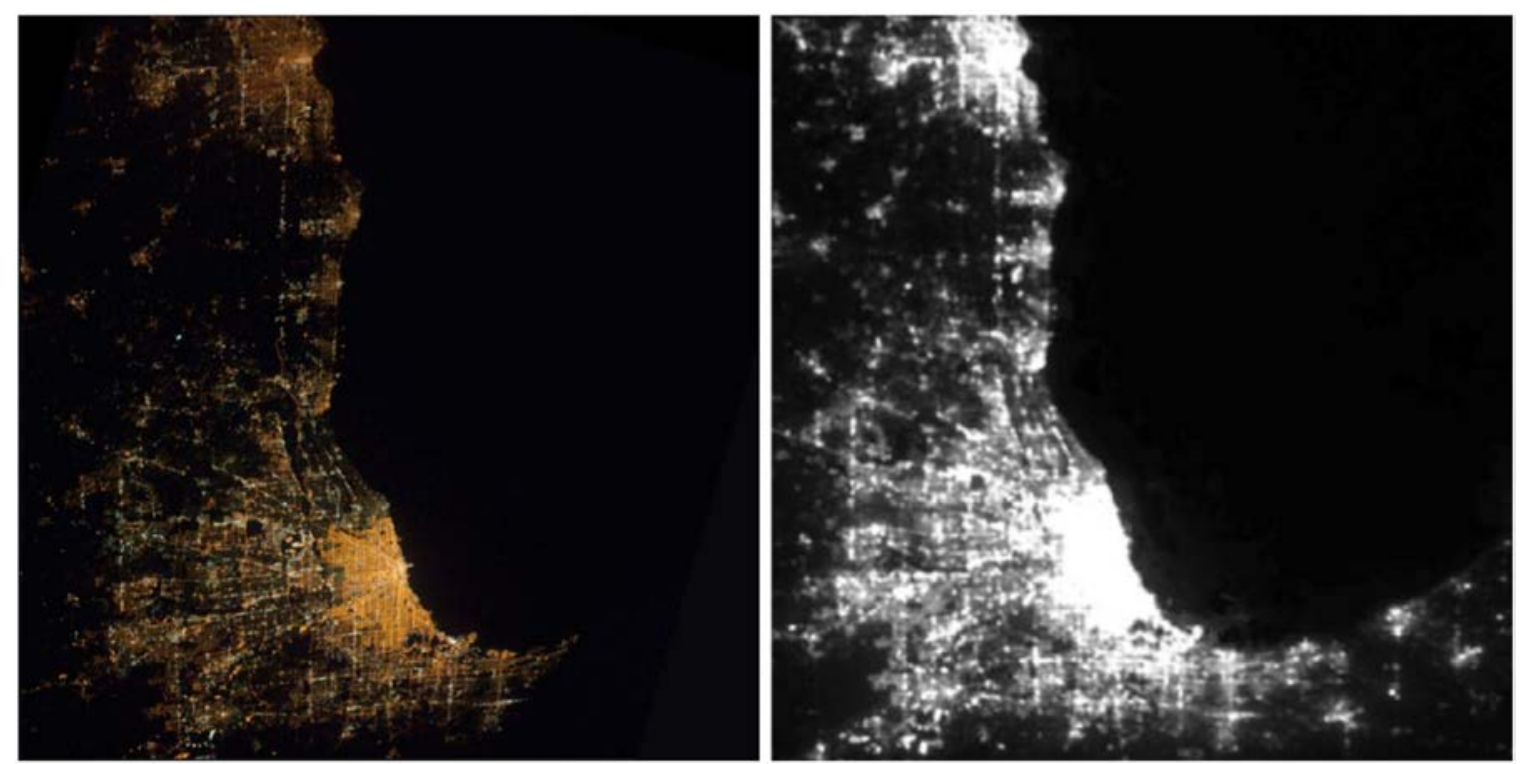

Fig. 2. Simulated NITESat (left) and actual VIIRS (right) images of the Chicago region. NITESat will provide a 7-fold increase in resolution over VIIRS as well as invaluable color data. NITESat simulation is a down-sampled image from the ISS. Image credits: Earth Science and Remote Sensing Unit, NASA Johnson Space Center and VIIRS Image and Data by NOAA's National Geophysical Data Center.

\subsection{Capture time-relevant data}

The mission's orbit will provide varied overpass times across the imaging footprint. This will allow the collection of night luminance data across varied times of the night. As night progresses many towns and businesses begin turning off or reducing the amount of lights they use. To capture an accurate understanding of the impact of LP, the optimal time of data collection is before 10pm local time when peak lighting can be observed [8]. Due to its varied overpass times, NITESat will be able to image areas during the height of nighttime illumination as well as being able to distinguish patterns in lighting change across night time hours. Additionally, NITESat is expected to be operational for more than a year. This will allow the analysis of seasonal changes or perhaps even monitoring the transitional changes in communities or businesses that change their lighting fixtures or lighting use for better or worse.

\subsection{Educational outreach and engagement}

A primary goal of the NITESat and GONet mission beyond research is educational. The Far Horizons program at the Adler Planetarium has engaged students and volunteers for over a decade with hands-on scientific and engineering projects. NITESat and GONet will leverage that experience in educational programming and training to facilitate the design, testing and operations of the mission.

Additionally, to implement the GONet array a program of LP education and awareness will be used as the basis of training for schools, clubs and communities who host the GONet stations. This offers the opportunity to not only have the public intimately involved in the science of the mission, but these same participants can help raise the profile of LP awareness in their communities. This public investment of effort can potentially turn into public advocacy for smart lighting practices. One could imagine a scenario such as students at a rural school, who host a GONet station, coordinating a town-wide "Lights Out Night" to compare NITESat and GONet data and demonstrate the effects of different lighting practices. 


\section{NITESat Design}

NITESat will be a $2 U(10 \times 10 \times 20 \mathrm{~cm})$ CubeSat based on a commercially available (GOMSpace) bus. It will include Commercial Off The Shelf (COTS) power, communications, attitude control and flight computer modules. The main payload will be a low-light capable imager based on the three color (RGB) PCO Edge 3.1 sCMOS camera and a fast $(\mathrm{FL}=1.4) 25 \mathrm{~mm}$ Lensation lens.

During each favorable night-time overpass, multiple rapid (20ms exposure at a 0.1 s cadence) images will be taken and stacked by an on-board processor. Stacked images will be stored and downlinked in losslessly compressed form. The target performance is given in Table 1.

Table 1. NITESat Imaging Parameters

\begin{tabular}{c|c|c|c|c}
\hline Resolution & Bands & Sensitivity & FOV & Dynamic Range \\
\hline $100 \mathrm{~m} / \mathrm{px}$ & $0.4-0.5,0.5-0.6,0.6-0.7 \mu \mathrm{m}$ & $<5 \times 10-9 \mathrm{~W} / \mathrm{cm} 2 / \mathrm{sr} / \mu \mathrm{m}$ & $30^{\circ}$ & $15 \mathrm{bits}$ \\
\hline
\end{tabular}

NITESat will orbit at an altitude of approximately $500 \mathrm{~km}$ with an inclination of $50^{\circ}$. Even accounting for cloud cover averages, our target region should be fully imaged multiple times in a year. In our reference orbit, imaging overpasses in the optimal twilight to midnight time frame occur in clusters lasting approximately 1 month separated by a 1 month period without imaging opportunities. This cadence allows the downlink of stored data during lulls in the imaging portion of the mission. The expected orbital lifetime of 1-2 years will allow the tracing of seasonal variations of the emission of artificial light at night.

\section{GONet Design}

The Ground Observing Network (GONet) is a complement to the satellite mission as well as an independent means of valuable data collection about localized light pollution. A primary component of the network is the GONet imager, which consists of a 5.0 Megapixel Point Grey Blackfly GigE Aptina MT9P006 camera equipped with a fisheye lens capable of capturing full $2 \pi$ sky images. The GONet imager is controlled using a Raspberry Pi 3 with images taken at regular intervals and processed for subsequent analysis.

The goal is to design a system requiring as little maintenance and the greatest ease of use as possible while still providing significant, useful data. The optimal system will be as autonomous as possible, allowing installation in relatively remote locations where power and data connectivity will not be provided. With these design goals some of the baseline features planned for the final instrument include:

- $\quad$ GPS for timing and location data

- Magnetometer for absolute orientation knowledge

- Weatherproof enclosure with automated articulated cover to reduce cleaning of the imaging aperture

- Solar charging power supply for long-term function with minimal maintenance

- Bluetooth connectivity to allow the easy upload of imaging schedules and download of stored data

Calibration is still a concern to be addressed. We are presently exploring a variety of approaches to calibrate the system with hardware and or software solutions. There is substantial research to inform potential solutions specifically for all sky monitoring systems using fisheye lenses [10]-[12]. In the current design iteration, the imaging routine includes the automatic capturing of calibration data such as dark frames and identification of stars with known brightness and sky positions. Figure 3 shows the current optical and electronic system. Development of proper packaging and field hardening is underway. 


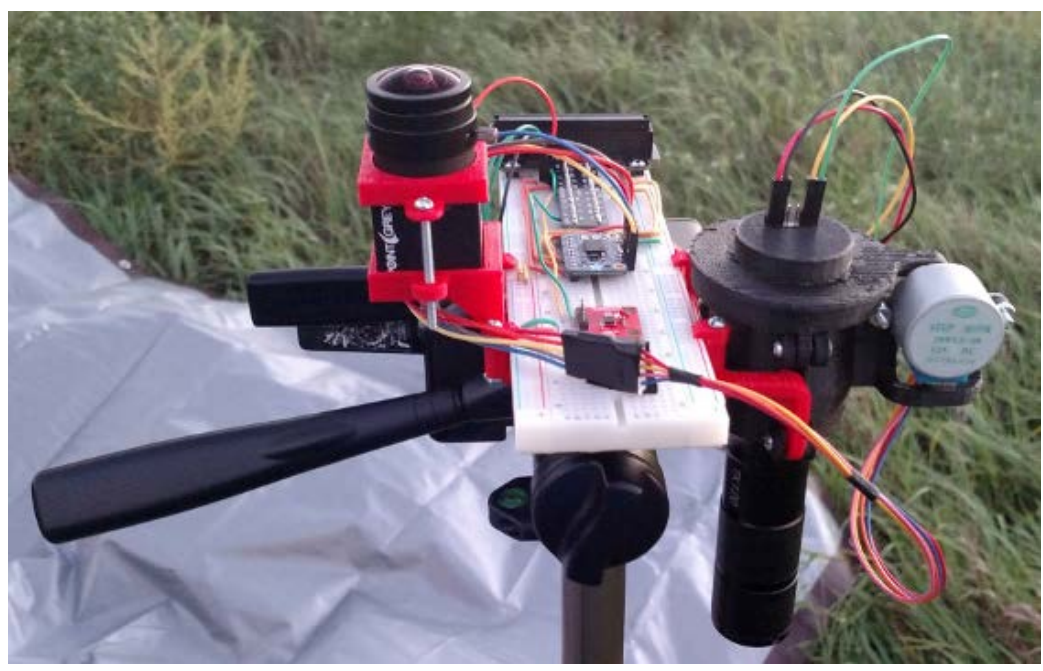

Fig. 3. Second GONet prototype used during September 10, 2016 test observations

\section{Preliminary Testing and Results}

An extensive qualifying program is currently underway using stratospheric high altitude balloon (HAB) flights and laboratory tests to ensure the main payload meets required specifications, is space-ready and both the hardware and software are fully integrated with the spacecraft bus. These tests used a representative imaging system, a Point Grey Grasshopper 2 with a 25mm f/1.4 lens, to confirm resolution and sensitivity assumptions. The flights also serve as a preliminary test of the mission operations.

Using HAB flights, we were able to conduct two validation tests with representative model hardware in 2016. A prototype GONet system was set up in a remote and relatively dark location along the predicted path of the HAB flight. For the first test flight, the GONet station used a Raspberry Pi 2 and was equipped with a Point Grey Firefly MV 13S2C-CS, a fisheye lens, GPS, and a calibrated light source to help confirm the sensitivity and resolution of the in-flight imaging system (see Fig. 4). The light source was periodically covered when GONet took images by using a stepper motor to place a $3 \mathrm{~d}$ printed cap over it, and a luminosity sensor in the cover was used to measure the intensity of the light source.

On the second test flight, the GONet Firefly camera was replaced by the Blackfly camera because of better $\mathrm{S} / \mathrm{N}$ with long exposures needed under dark conditions. Both hardware and software improvements were made to increase the accuracy of data collection and system efficiency. A magnetometer was added to detect the orientation of the images, and a python script was created to automate magnetometer calibration which can be quickly performed at the observing site. Initial test results have indicated the system can collect data able to meet the mission requirements. (see Fig. 5) 


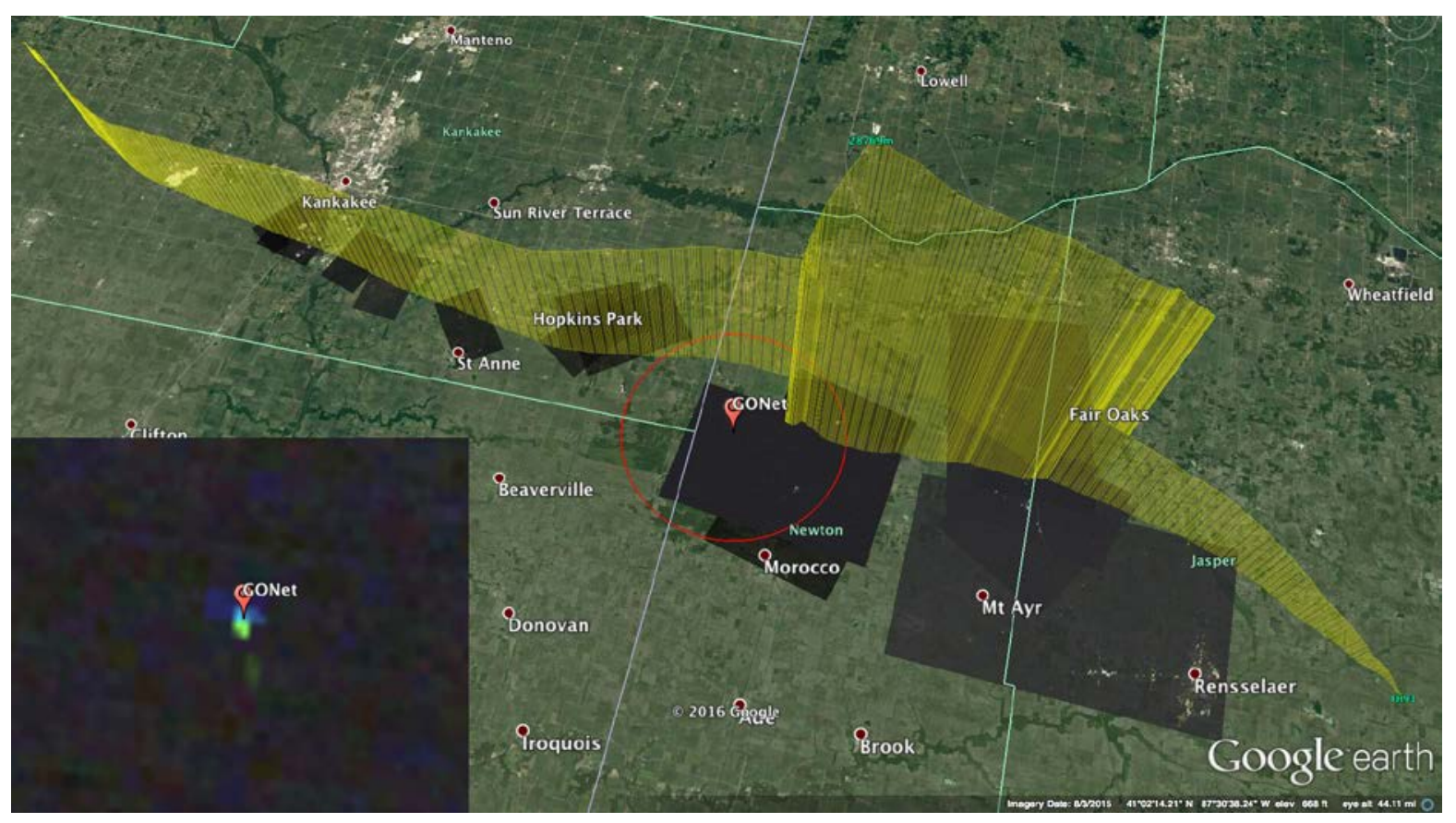

Fig. 4. Results of first HAB NITELITE/GONet test flight showing flight path and select the images from flight. Inset: Identification of the GONet calibration light in an image from an altitude of 28 kilometers.
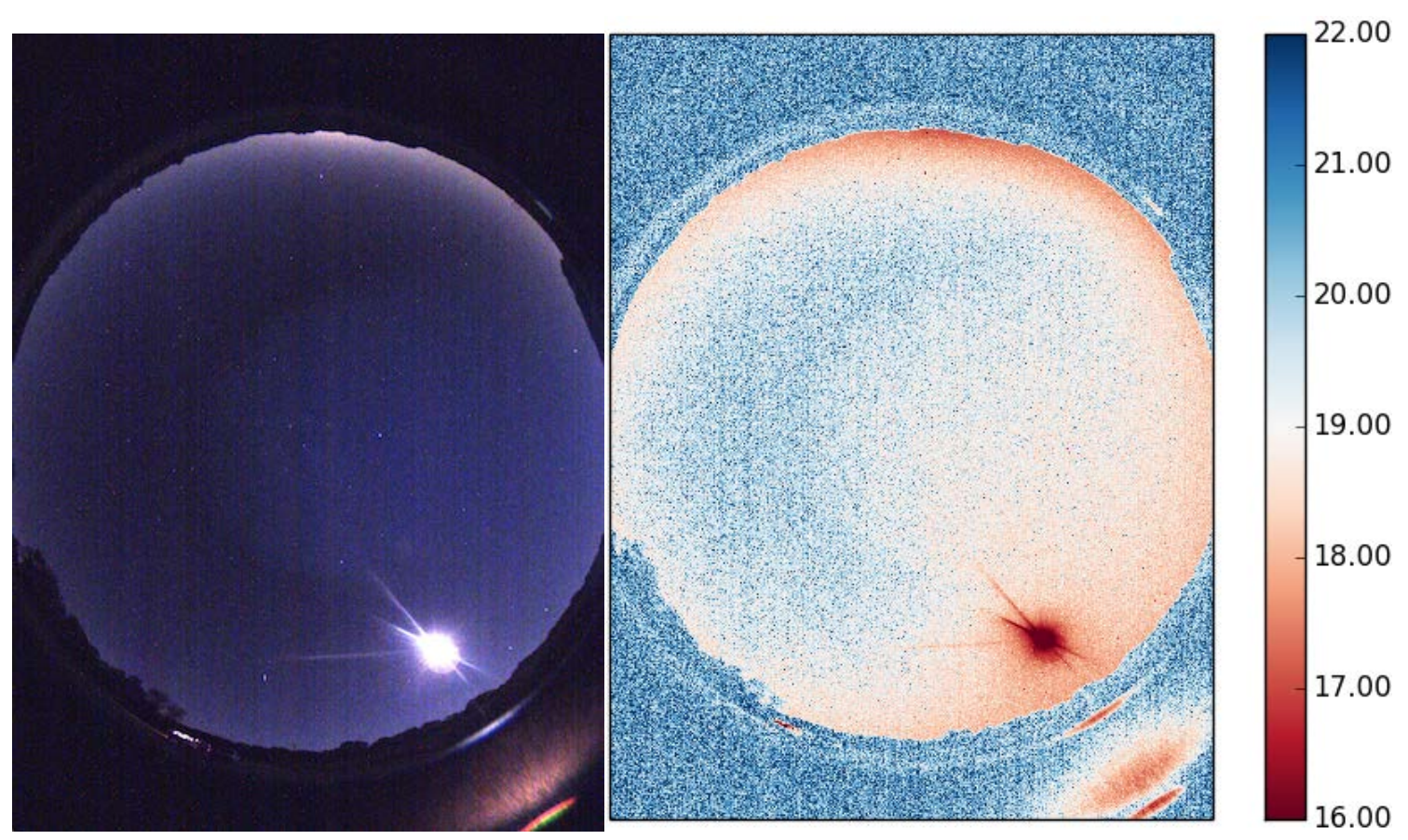

Fig. 5. A single sky image (left) taken with a 3.5sec exposure by the second GONet prototype. Dark frames are used to remove systematic noise. The resulting images are calibrated to mag/arcsec2 as shown in the heat map (right). A quarter phase moon is prominent in the image. 
The GONet test results have helped guide the design of the next version of the system including optimization of the exposure range, addition of in-image metadata and software improvements for simplification. Information from the test flights resulted in a third iteration of the software package. The latest software update makes the GONet a fully autonomous system capable of significantly reducing noise, determining accurate brightness measurements of light sources, and producing both quantitative and qualitative visualizations of statistical data.

\section{Conclusion}

Small, single-purposed nanosatellites (CubeSats) have the potential to provide valuable data for light pollution research. The NITESat mission has been designed to take advantage of the relative low cost and simplicity of the CubeSat form factor while carefully maintaining the quality of the data collected. The mission requirements of NITESat and GONet have been specifically generated to demonstrate the feasibility of missions of this scale for research.

The Far Horizon team intends to make its data publically available in reduced form. We are also interested in collaboration with researchers in the light pollution community, education outreach professionals and engineers to help optimize our mission design and provide the most valuable data to best serve the community's goals. Partnerships with groups interested in providing additional downlink services, i.e., communication Ground Stations, may allow additional imaging opportunities beyond the mission target region.

\section{References}

[1] Blask, D. E., Brainard, G. C., Dauchy, R. T., Hanifin, J. P., Davidson, L. K., Krause, J. A., Sauer, L. A., Rivera-Bermudez, M. A., Dubocovich,M. L., Jasser, S. A., Lynch, D. T., Rollag, M. D., \& Zalatan F. (2005). Melatonin-depleted blood from premenopausal women exposed to light at night stimulates growth of human breast cancer xenografts in nude rats. Cancer research, 65(23), 11174-11184.

[2]Longcore, T., \& Rich, C. (2004). Ecological light pollution. Frontiers in Ecology and the Environment, 2(4), 191-198.

[3]Falchi, F., Cinzano, P., Duriscoe, D., Kyba, C. C. M., Elvidge, C. D., Baugh,K., Portnov,B. A., Rybnikova, N. A., \& Furgoni, R. (2016). The new world atlas of artificial night sky brightness. Science advances, 2(6), e1600377.

[4]Carhart, D. (2016). Chicago: A Study in Outdoor Lighting Practices and Costs. illinoislighting.org, Online available: http://www.illinoislighting.org/chicago.html. (Accessed: 23-Nov-2016).

[5]Steinbach, R., Perkins, C., Tompson,L., Johnson, S., Armstrong, B., Green, J., Grundy, C., Wilkinson, P., \& Edwards, P. (2015). The effect of reduced street lighting on road casualties and crime in England and Wales: controlled interrupted time series analysis. Journal of epidemiology and community health, jech-2015.

[6]Elvidge, C. D., Erwin, E. H., Baugh, K. E., Ziskin, D., Tuttle, B. T., Ghosh, T., \& Sutton, P. C. (2009, May). Overview of DMSP nightime lights and future possibilities. In Urban remote sensing event, 2009 Joint (pp. 1-5). IEEE.

[7]Huang, Q., Yang, X., Gao, B., Yang, Y., \& Zhao, Y. (2014). Application of DMSP/OLS nighttime light images: A meta-analysis and a systematic literature review. Remote Sensing, 6(8), 6844-6866.

[8] Elvidge, C. D., Baugh, K. E., Zhizhin, M., \& Hsu, F. C. (2013). Why VIIRS data are superior to DMSP for mapping nighttime lights. Proceedings of the Asia-Pacific Advanced Network, 35, 62-69.

[9]Elvidge, C. D., Keith, D. M., Tuttle, B. T., \& Baugh, K. E. (2010). Spectral identification of lighting type and character. Sensors, 10(4), 3961-3988.

[10]Rabaza, O., Aznar-Dols, F., Mercado-Vargas, M. J., \& Espín-Estrella, A. (2014). A new method of measuring and monitoring light pollution in the night sky. Lighting Research \& Technology, 46(1), 5-19.

[11] Nievas Rosillo, M. (2013). Absolute photometry and Night Sky Brightness with all-sky cameras. Máster Interuniversitario en Astrofísica UCM-UAM.

[12] Román, R., Antón, M., Cazorla, A., De Miguel, A., Olmo, F. J., Bilbao, J., \& Alados-Arboledas, L. (2012). Calibration of an all-sky camera for obtaining sky radiance at three wavelengths. Atmospheric Measurement Techniques, 5(8), 2013. -2024. 